\title{
Prevalence of sensitization to specific allergens in allergic patients in Beijing, China: A 7-year retrospective study
}

\author{
Ying Li, ${ }^{1}$ Yusan An, ${ }^{1}$ Yun Hao, ${ }^{1}$ Luo Zhang, ${ }^{1,2}$ Yuhui Ouyang ${ }^{2}$
}

\begin{abstract}
Background: Knowledge of the prevalence of common sensitizing allergens may aid in overall management of allergic disease in a specified area.

Objective: The aim of this study was to identify and analyse the prevalence of common inhaled and food sensitizing allergens in Beijing.

Methods: This was a retrospective study, analysing demographic data and serum sIgE antibody test results from 59057 outpatients who presented to Beijing TongRen Hospital, from January 2013 to December 2019.

Results: 28879 patients (48.9\%) showed positive sIgE test results; with significantly more males aged under 16 years sensitized to at least one allergen than females, and most patients (53.62\%) were sensitized to multiple allergens. The first inhaled sensitizing allergens was Artemisia grass (11910 (41.24\%)); and the first food allergens was crab (3547 (12.28\%)). For Artemisia sensitized patients, sIgE levels were mostly at level 5. The number of patients with ragweed allergy is increasing year by year. The detection rates for sIgE to Artemisia, common ragweed, and Humulus grass allergens were significantly higher in August and September. R package ggplot2 analysis, demonstrated strong correlations between tree allergens and common ragweed and Humulus grass allergens (phi coefficients $=0.50$ and 0.46 , respectively; both $P<0.01)$.
\end{abstract}

Conclusion: The prevalence of sensitization to different allergens in Beijing showed Artemisia grass was the most commonly inhaled sensitizing allergen, and the number of patients with ragweed grass allergy was increasing by year.

Key words: Inhaled allergens, food allergens, serum specific IgE, R package ggplot2, Chinese patients, sensitization prevalence, Artemisia, dust mites, crab, peanut

From:

${ }^{1}$ Department of Otolaryngology Head and Neck Surgery,

Beijing TongRen Hospital, Capital Medical University, Beijing, China

${ }^{2}$ Department of Allergy, Beijing TongRen Hospital,

Capital Medical University, Beijing, China

\section{Introduction}

Allergic disease is a group of common of immune-mediated disorders; including allergic rhinitis (AR), asthma, allergic conjunctivitis, atopic dermatitis (AD), and food allergy; which are mainly caused by an immunoglobulin E (IgE)-dependent immunological reaction to an innocuous environmental antigen (allergen) according to the site of contact with the allergen. Diagnostic tests are based on the demonstration of allergen-specific IgE in the skin (skin prick tests) or in the serum

\section{Corresponding author:}

Yuhui Ouyang

Department of Allergy, Beijing TongRen Hospital,

Capital Medical University,

No. 1, Dongjiaominxiang, Dongcheng District, Beijing 100730, China E-mail: oyyuhui@sina.com

(serum specific IgE test), and the immunoblot assay is most commonly used for semi-quantitative detection of the allergen/s. Allergic disease is continually rising worldwide, ${ }^{1}$ and is a common health problem that affects a substantial number of individuals worldwide; often negatively impacting the quality of life of the affected individual. AR is a common allergic disease of the nasal mucosa that affects around 10 to $40 \%$ of the population worldwide. ${ }^{2,3} \mathrm{~A}$ recent study from China has 
demonstrated that the self-reported prevalence rate of $A R$ in 18 major cities in China is $17.6 \% .^{4}$

Allergic diseases are closely related to demographic and environmental factors, and immune dysfunction. ${ }^{5-7}$ Although genetic, gender, age, and obesity are major factors that lead to the development of allergic diseases; ${ }^{8}$ information on the prevalence of the common allergens that trigger allergic disease may additionally provide a clinical rational for better overall management of allergic diseases in any specified area. Thus, the aim of the present study was to determine the prevalence of common inhaled and food allergens in Beijing, China, employing a retrospective analysis of data obtained for sIgE antibody testing over a period of past seven years in Beijing.

\section{Materials and Methods Patients}

This study included allergic patients who had presented to the Department of Allergy in Beijing TongRen Hospital between January 2013 to December 2019. Patients with symptoms such as sneezing, nasal itching, runny nose and nasal congestion, or patients with symptoms such as cough, chest tightness and wheezing suspected allergic rhinitis or asthma, and patients with itching and rash suspected atopic dermatitis were recruited continuously in Allergy Clinic of Beijing Tongren Hospital, by which the sensitization to relevant allergens was assessed by sIgE. All of the patients had resided in Beijing for at least 1 year and undergone allergy testing for specific allergens when attending the Department of Allergy in Beijing TongRen Hospital. Patients not from Beijing or with nasal infections or tumors were excluded. Patients with drug allergies were excluded. The present study was conducted in accordance with the Declaration of Helsinki and approved by the Medical Ethics Committee of Beijing TongRen Hospital (TRECKY2020-076).

\section{Serum sIgE test}

Blood samples collected from each of the 59057 patients was analysed for sIgE using the AllergyScreen test (EUROBlotOne, Beijing, China), an immunoblot assay used to semi-quantitatively detect circulating allergen-specific immunoglobulin E (IgE) in human serum. The sIgE antibody level was evaluated using a panel of the most prevalent local inhalant and food allergens; of which the common inhaled allergens included grass pollen (including Humulus, Artemisia, common ragweed), mold (including penicillium aspergillus, herb bud branch mold, smoke Aspergillus and Alternaria), blattella, animal dander (including cat and dog), house dusts, dust mites (including Dermatophagoides pteronyssinus and Dermatophagoides farinae), and tree pollen (including Salix babylonica, Populus, Ulmus pumila); and the common food allergens included crab, shrimp/prawn, marine fishes (cod, lobster, scallops), lamb, beef, soybean, peanut, cow's milk, and egg.
A concentration of any sIgE antibody at least $0.35 \mathrm{IU} /$ $\mathrm{mL}$ was considered positive sensitization. Based on the concentration of the antibody detected, the semi-quantitative test results of the sIgE was classified into one of the following six allergic levels: level 0: $>0.35 \mathrm{IU} / \mathrm{mL}$; level 1: 0.35-0.69 IU/ $\mathrm{mL}$; level 2: 0.7-3.49 IU/mL; level 3: 3.50-17.49 IU/mL; level 4: 17.50-49.99 IU/mL; level 5: 50.00-100.00 IU/mL; level 6: $>100.00 \mathrm{IU} / \mathrm{ml}$. $^{9}$

\section{Statistical analysis}

Statistical analyses were performed using the SPSS 25.0 statistical software (IBM Co., Armonk, NY, USA). Significance of differences in gender and ages was compared using the Chi-square test and correlations between serum sIgE and age were analysed by Spearman's rank-order test. Values of $P$ $<0.05$ were considered to be statistically significant.

Sector graphs and correlation analysis were performed using GraphPad Prism 7.0 software (GraphPad Software Inc., La Jolla, CA, USA). The heatmap of the association between two allergens was illustrated using an $\mathrm{R}$ package ggplot2. Phi coefficient was applied to measure the correlation between two allergens in all sIgE-positive patients; with values of Phi coefficient $\geq 0.70$ considered to be a very strong correlation, between 0.40 and 0.69 considered to be a strong correlation, between $0.30-0.39$ considered to be moderate correlation, between $0.16-0.29$ considered to be weak correlation, and $\leq 0.15$ considered to be negligible correlation.

\section{Results \\ Characteristics of the study population}

A total of 59057 patients had undergone allergy testing for specific allergens during the period from January 2013 to December 2019. The age of the patients ranged from 1-95 years (mean \pm SD: $32.60 \pm 6.26$ ), and the patients were therefore grouped according to nine age groups; including $\leq 5$ years, 6-10 years, 11-15 years, 16-20 years, 21-30 years, 31-40 years, 41-50 years, $51-60$ years, and $\geq 61$ years. Assessment of data from these patients demonstrated that 28879 (48.90\%) patients had positive $\mathrm{sIgE}$ test results (i.e. $0.35 \mathrm{IU} / \mathrm{mL}$ to $>100$ $\mathrm{IU} / \mathrm{ml})$, and $30178(51.09 \%)$ negative sIgE test results $(<0.35$ $\mathrm{IU} / \mathrm{mL})$. Among the patients with positive $\mathrm{sIgE}$ test results, 15391 (53.29\%) patients were male, and 13488 (46.71\%) patients were female. Analysis of gender differences among patients who tested positive for sIgE in each age group demonstrated that significantly greater numbers of males in age groups $\leq 5$ years, 6-10 years and 11-15 years, had positive sIgE levels than females $\left(\chi^{2}=23.03, \chi^{2}=18.23, \chi^{2}=12.24 ; P\right.$ $<0.01, P<0.01, P<0.01$, respectively). However, no gender difference was detected in patients aged over 15-years (Supplementary Table1). Similarly, analysis of allergen-positivity by age demonstrated significantly greater numbers of patients aged 21-30 years and 31-40 years to be sIgE positive than patients in other age groups $(P<0.01, P<0.01)$. 


$$
50
$$

$$
45
$$$$
40
$$

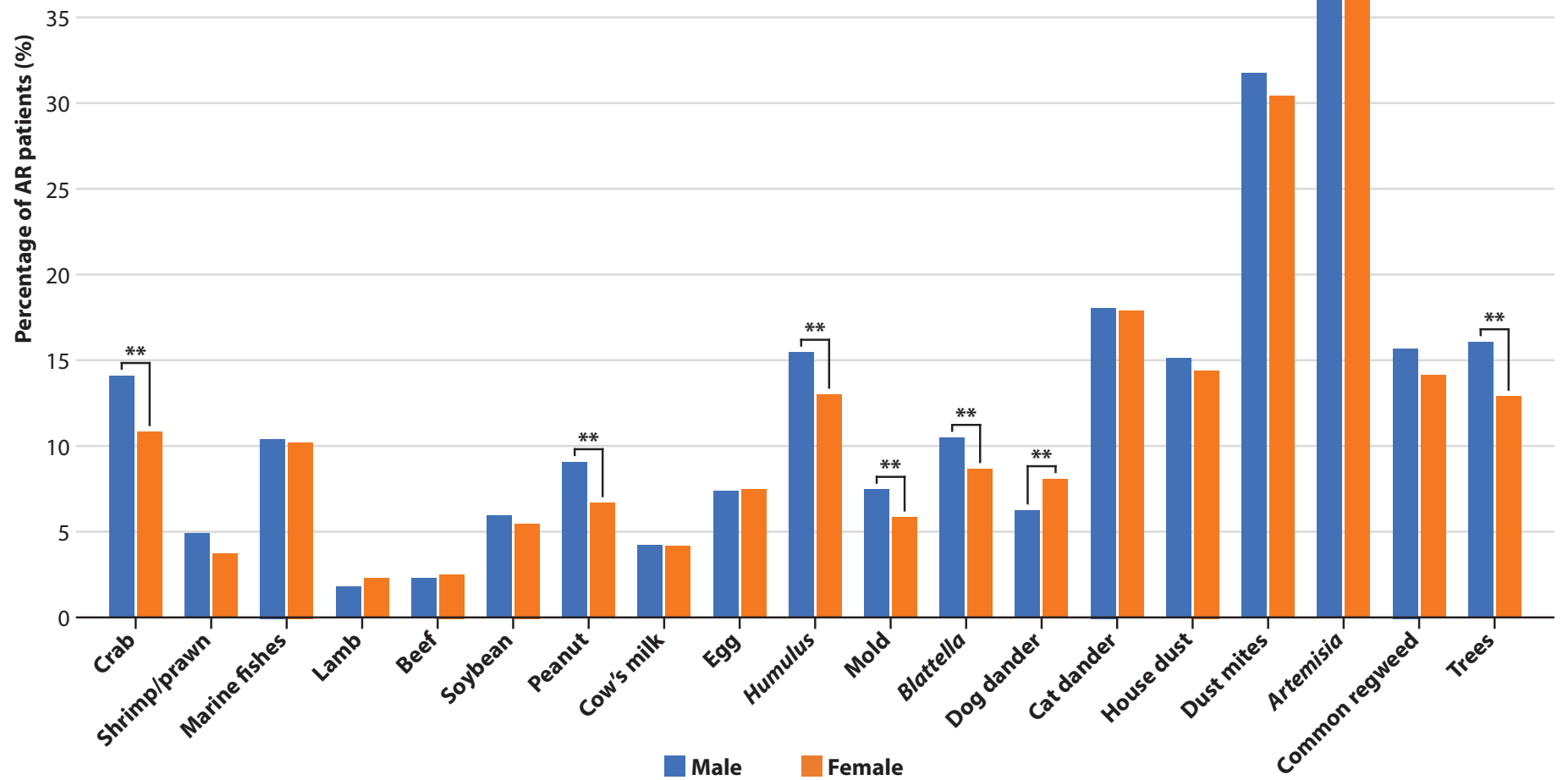

Figure 1. The percentage of total patients sensitized to specific allergens. Blue bar diagram shows male allergen-positive patients and Red bar diagram shows female allergen-positive patients. $P$ value was calculated using the chi-squared test. ${ }^{*} p<$ $0.05,{ }^{* *} p<0.01$.

\section{Patients with inhaled-and food allergen-sensitization in dif- ferent gender/age groups}

The distribution of patients sensitized to specific allergen is shown in Figure 1. Overall, 25077 cases (86.83\%) showed positive sIgE to inhaled allergens and 10470 cases (36.25\%) showed positive sIgE to food allergens. The top six inhaled allergens among patients were Artemisia grass (11910, 41.24\%), dust mites $(8921,30.89 \%)$, cat dander $(5114,17.71 \%)$, common ragweed grass $(4239,14.68 \%)$, house dust $(4190,14.51 \%)$ and trees $(4134,14.31 \%)$; and the top three food allergens were $\mathrm{crab}(3547,12.28 \%)$, marine fishes $(2895,10.02 \%)$, and peanut (2216, 7.67\%) (Figure 1).

Significant gender differences were found in patients with sensitivity to six allergens; with more males than females being sensitized to $\mathrm{crab}\left(\chi^{2}=71.69, P<0.01\right)$, trees $\left(\chi^{2}=60.41\right.$, $P<0.01)$, peanut $\left(\chi^{2}=58.76, P<0.01\right)$, Humulus grass $\left(\chi^{2}\right.$ $=35.54, P<0.01)$, mold $\left(\chi^{2}=30.94, P<0.01\right)$, and Blattella $\left(\chi^{2}=28.43, P<0.01\right)$. In contrast, significantly higher number of females were sensitised to only dog dander compared to males $\left(\chi^{2}=30.94, P<0.01\right)$ (Figure 1).
The distribution of allergen-positive patients in different age groups is shown in Figure 2. The highest percentage of house dust sIgE-positive patients were mainly distributed in age groups 11-15 years and 16-20 years (Figure 2A); whereas mold-sensitized patients were significantly higher in age groups $<5$ years and 6-10 years, compared to other groups $(P$ $<0.01, P<0.01$, respectively, Figure 2B). In the case of food allergens, the percentage of cow's milk and egg-sensitized patients were mainly distributed in the age group $<5$ years old, and progressively decrease with increasing age (Figure 2C and 2D). In contrast, the percentage of patients sensitized to crab and marine fishes showed an increasing trend with age after age six (crab: male $\mathrm{R}=0.97, P<0.01$ and female $\mathrm{R}=$ $0.97, P<0.01$; marine fishes: male $\mathrm{R}=0.98, P<0.01$ and female $\mathrm{R}=0.90, P<0.01$, respectively) (Figure $2 \mathrm{E}$ and $2 \mathrm{~F}$ ). However, no age-specific distribution was noted for any other allergen. 
A

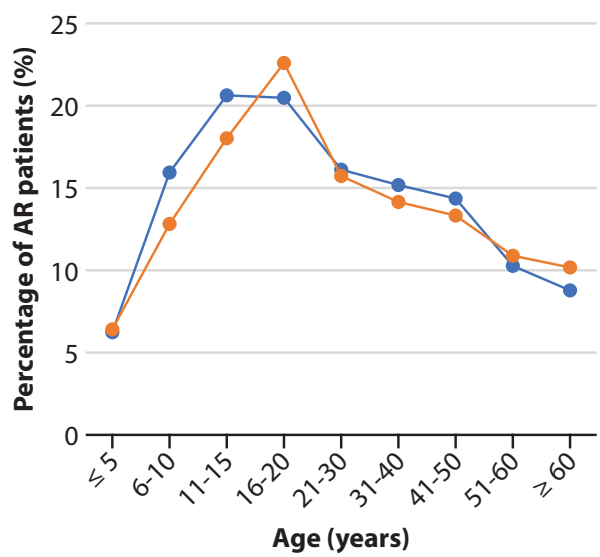

$\rightarrow$ House dust (Male) $\rightarrow$ House dust (Female)

D

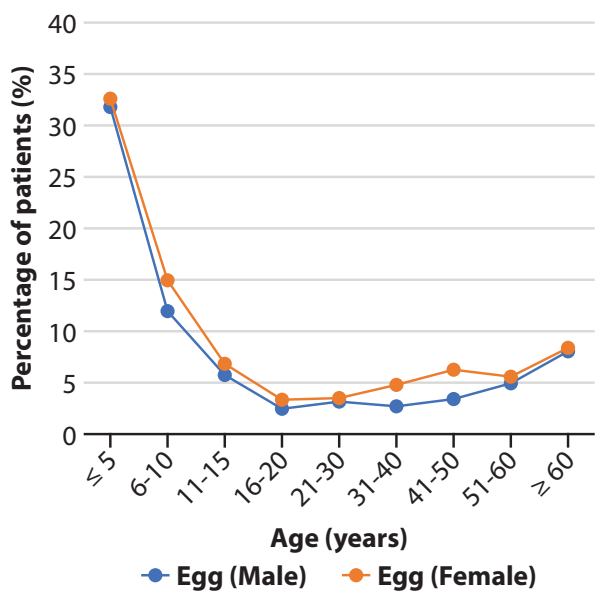

B

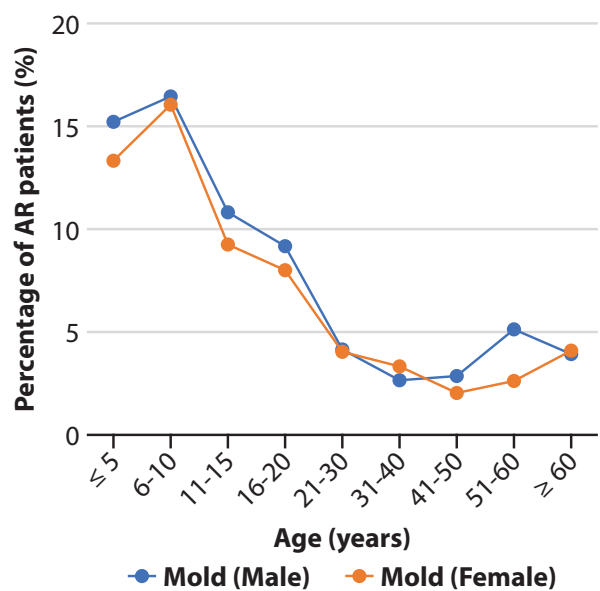

E

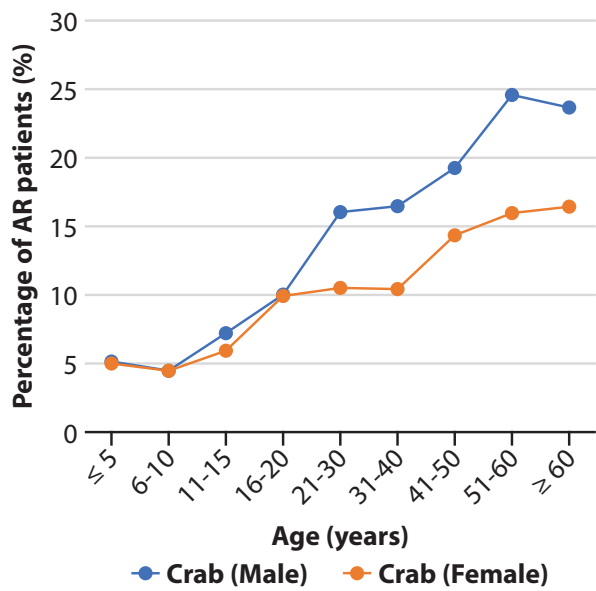

C

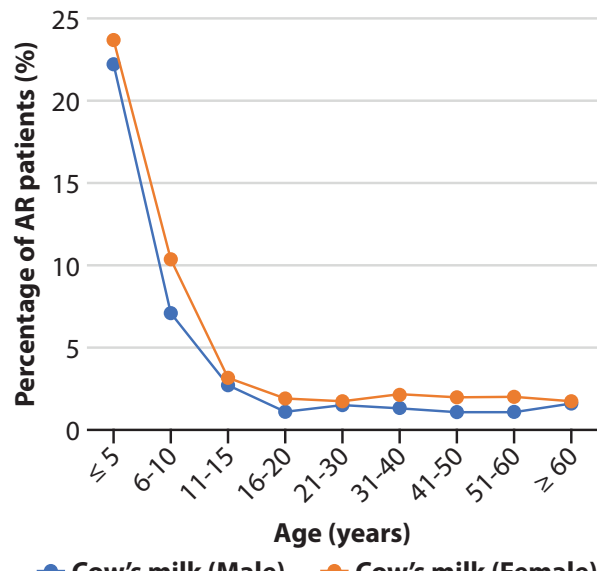

- Cow's milk (Male) - Cow's milk (Female)

$\mathbf{F}$

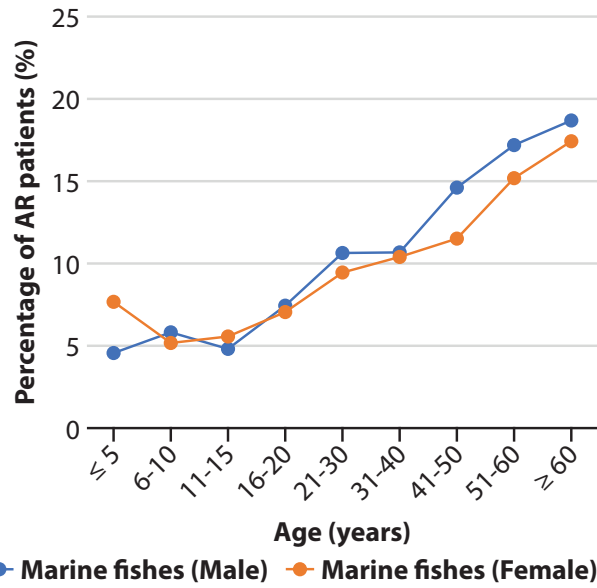

Figure 2. Percentage of patients positive to specific allergens in different age groups. (A) house dust, (B) mold, (C) crab, (D) marine fishes, (E) cow's milk, (F) egg. Blue line, male patients; Red line, female patients. The correlations between serum sIgE and age were analysed by Spearman's rank-order test.

A

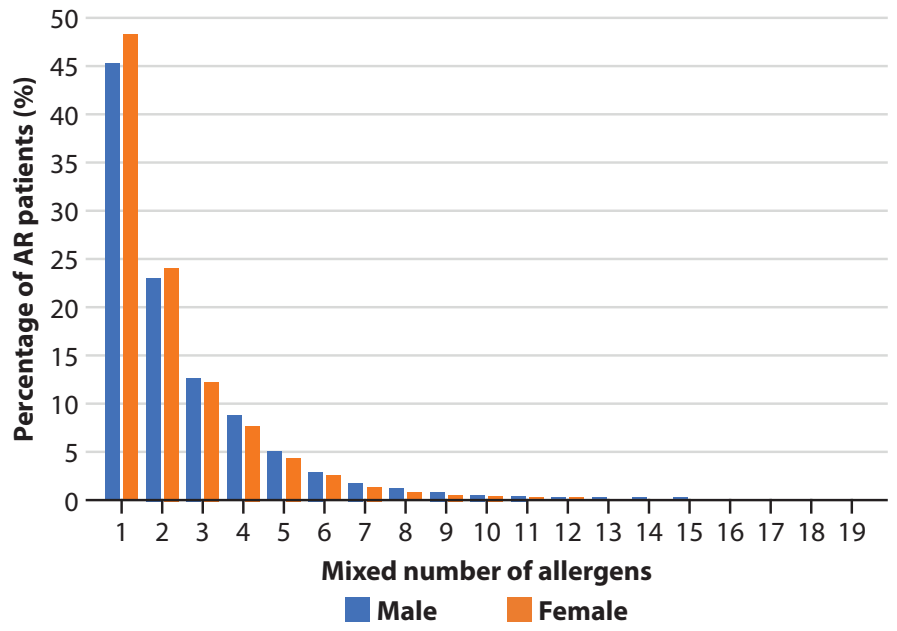

B

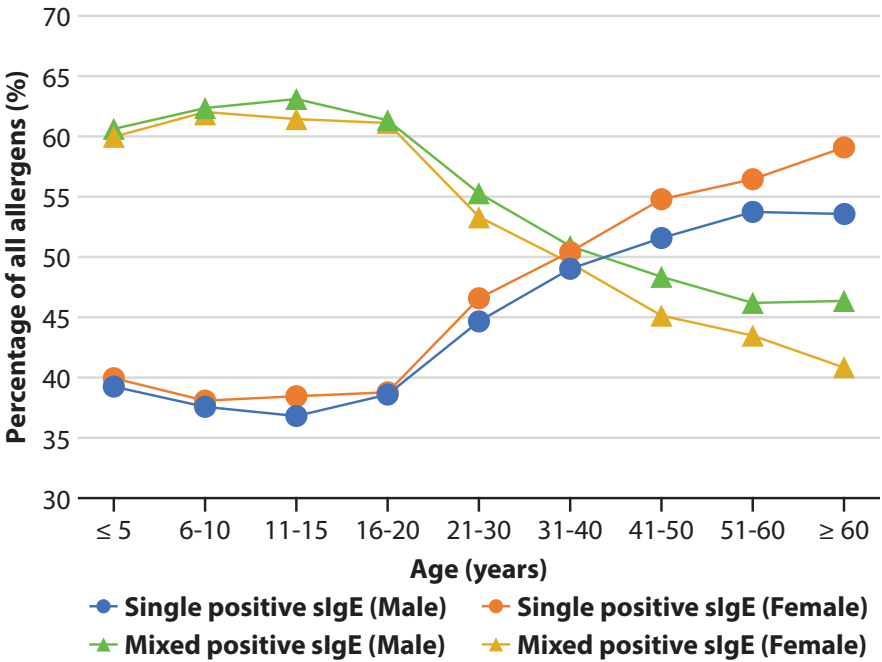

Figure 3. Distribution of patients sensitized to one or more allergens. (A) Percentage of patients with sIgE to different numbers of allergens. Blue bar diagram, male patients; Red bar diagram, female patients. $P$ value was calculated using the chisquared test. (B) Percentage of patients with sIgE to single and multiple allergens in different age groups. Blue line, male patients with single positive allergen; Green line, male patients with multiple positive allergens; Red line, female patients with single positive allergen; Yellow line, female patients with multiple positive allergens. ${ }^{*} p<0.05,{ }^{* *} p<0.01$. 


\section{Patients with single/multiple positive allergens}

Assessment of the number of different allergens the patients were sensitized to showed that $46.38 \%$ patients (male $44.98 \%$, female $47.98 \%$ ) were sensitized to a single allergen, and $53.62 \%$ patients were sensitized to multiple allergens (Figure 3A).

The proportion of patients with multiple allergens sensitivity was higher than that of patients with single allergen before 31-40 years old, but after 31-40 years old the proportion of patients with single allergen was higher than that of patients with multiple allergens (Figure 3B). Moreover, the proportion of patients with multiple allergens decreased gradually from age 21-30 years (male $\mathrm{R}=0.95, P<0.01$; female $\mathrm{R}=0.97, P<$ 0.01); whereas the proportion of patients with single allergens are opposite (male $\mathrm{R}=-0.95, P<0.01$; female $\mathrm{R}=-0.97, P<$ 0.01) (Figure 3B).

\section{sIgE levels of different allergens}

Analysis of the level of sensitization to specific allergens demonstrated that patients with positive food allergens and house dusts, mold, Blattella, common ragweed and trees allergens mainly presented with sIgE at levels 1 and 2; whereas Artemisia grass allergen-positive patients mainly presented with sIgE at level 5 (Figure 4).

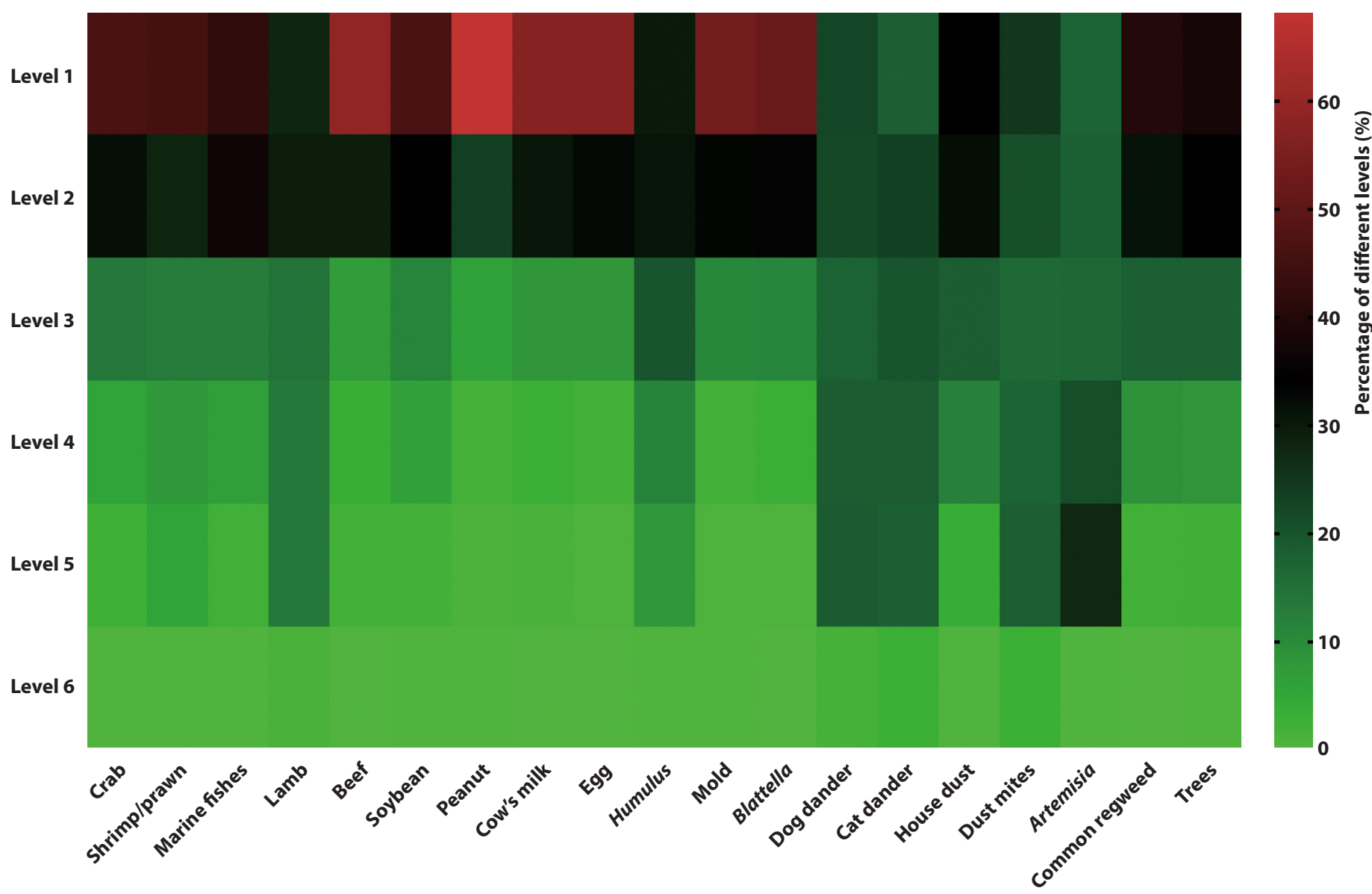

Figure 4. The level of sIgE detected in different allergen-positive patients. Percentage of patients with sIgE levels are shown according to a specific color chart; with a large percentage displayed in red, small percentage displayed in green, and color intensity proportional to the percentage. 


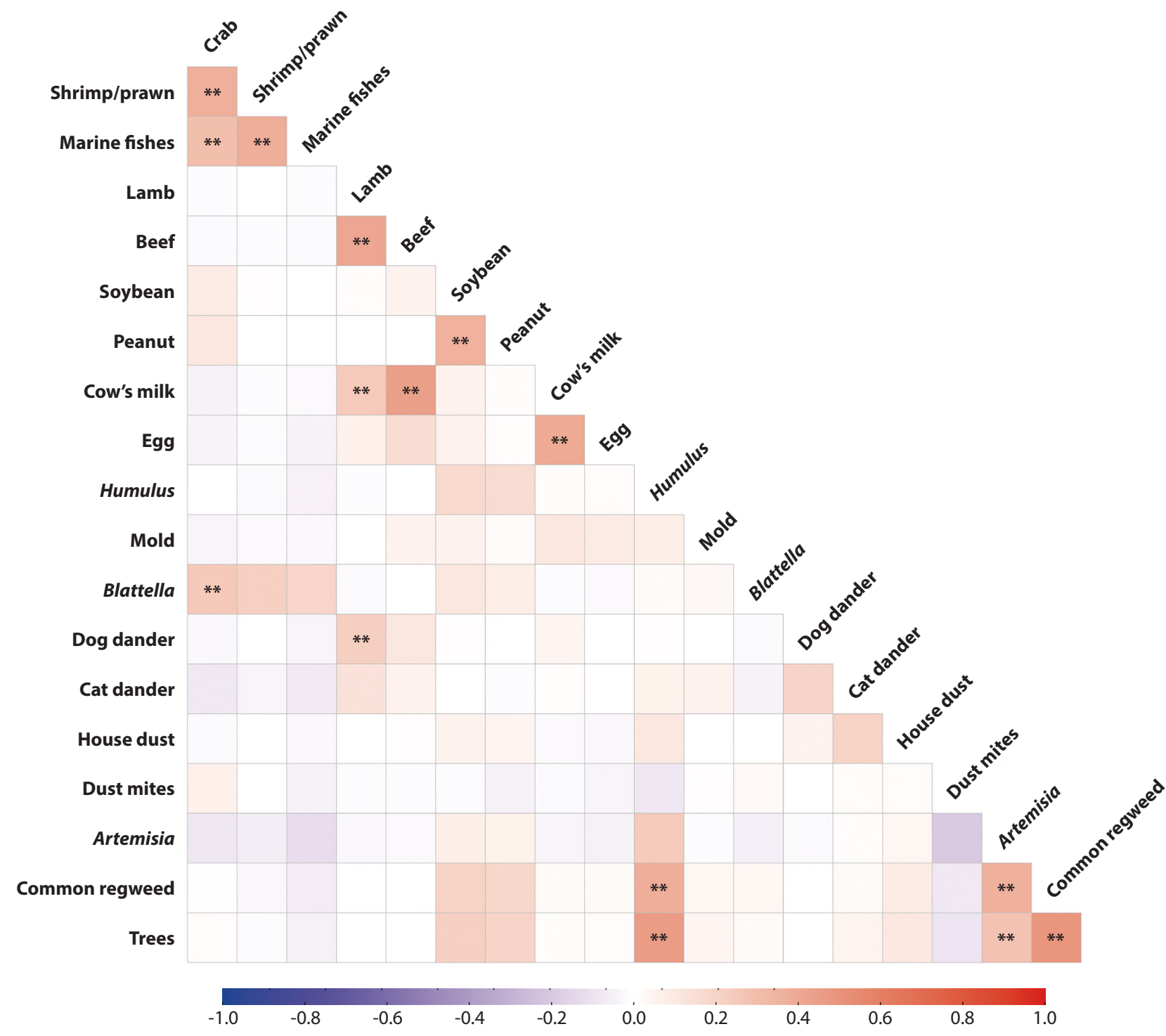

Figure 5. Correlations between allergens. The heatmap is illustrated using an $R$ package ggplot2. Phi coefficient is expressed as a color chart; with positive correlations displayed in red, negative correlations displayed in blue, and color intensity proportional to the correlation coefficients. Phi coefficient $\geq 0.70$ was considered a very strong correlation, $0.40-0.69$ a strong correlation, 0.30-0.39 a moderate correlation, $0.16-0.29$ a weak correlation, and $\leq 0.15$ a negligible correlation. ${ }^{\star} p<0.05 ;{ }^{\star *} p$ $<0.01$.

\section{Correlation between allergens}

The association between two allergens was analysed using an R package ggplot2 and Phi coefficient was applied to measure the correlation between two allergens in 28879 sIgE-positive patients. In the case of inhaled allergens, trees and common ragweed/Humulus grass exhibited a strong correlation (phi coefficients 0.50 and 0.46 , respectively; both $P<0.01$ ) (Figure 5). Similarly, beef and cow's milk, beef and lamb, and cow's milk and egg allergens were strongly correlated positively (phi coefficients were $0.45,0.41$, and 0.40 , respectively; both $P<0.001$ ) (Figure 5). Additionally, common ragweed and Humulus, common ragweed and Artemisia, Crab and Marine fishes, crab and shrimp/prawn, and Soybean and Peanut also showed moderate positive associations (phi coefficients were $0.38,0.38,0.37,0.37$ and 0.36 respectively; all $P<0.01$ ) (Figure 5), while crab and Blattella showed a weak association (phi coefficient was $0.26 ; P<0.01$ ) (Figure 5).

\section{Annual and seasonality of allergen sensitivity}

We analysed the variation of positive detection rates of different allergens in different years and months. The percentage of patients sensitized to common ragweed showed an increasing trend year by year (male $\mathrm{R}=0.96, P<0.01$ and female $\mathrm{R}$ $=0.97, P<0.01)$ (Figure 6A). The positive detection rates for sIgE to Artemisia, common ragweed, and Humulus grass allergens were significantly higher in August and September than in other months $(P<0.01)$ (Figure 6B, C, and D). Similarly, the sIgE detection rate for house dust was significantly higher in May than in other months $(P<0.01)$ (Figure 6E) and the positive detection rate for mold sIgE was significantly higher in January, February and July than in other months $(P<0.01)$ (Figure 6F). No statistically significant seasonal differences were detected for any of the food allergens. 
A
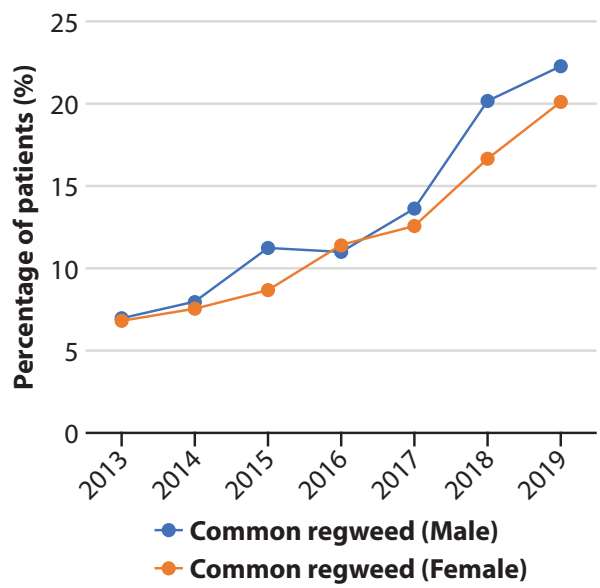

D

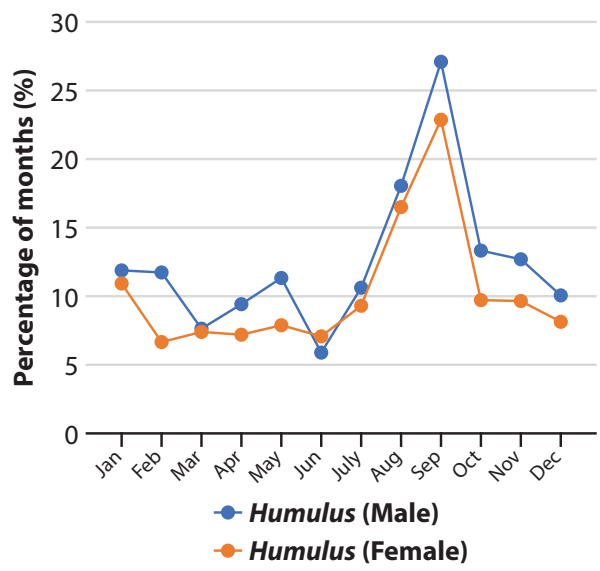

B

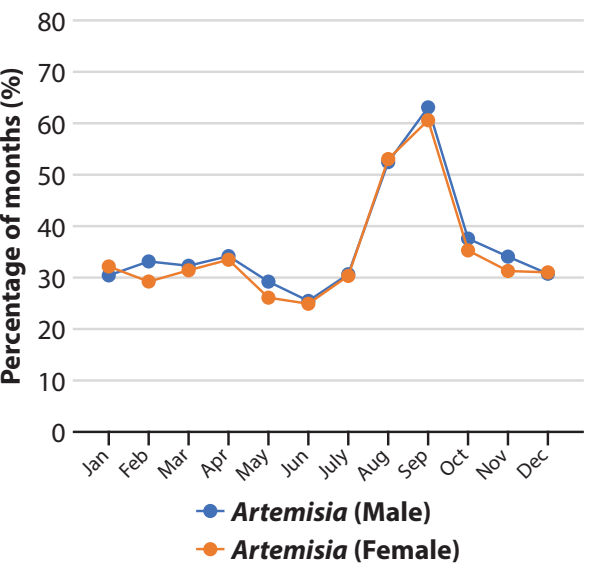

E

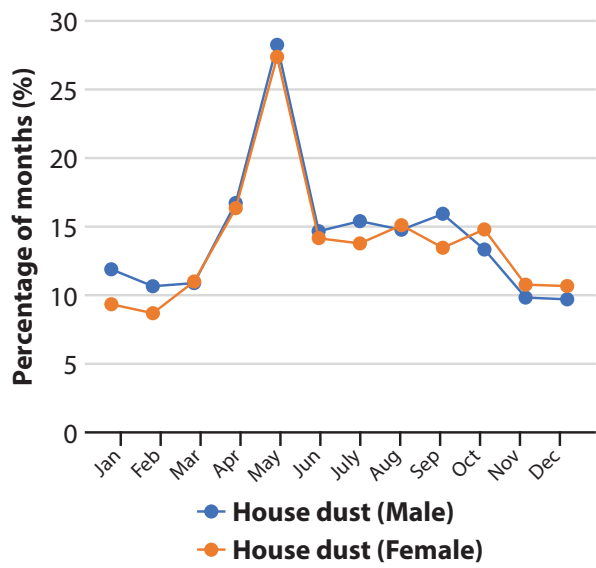

C 30

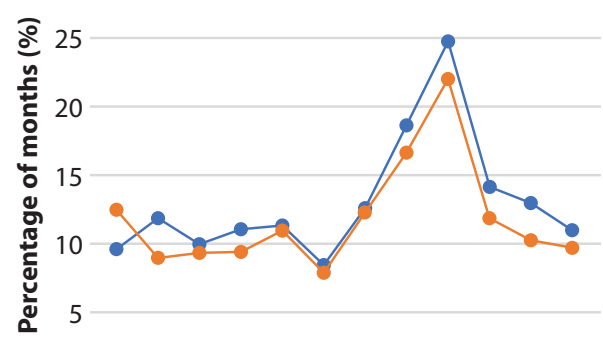

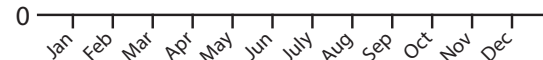

- Common regweed (Male)

- Common regweed (Female)

$\mathbf{F}$
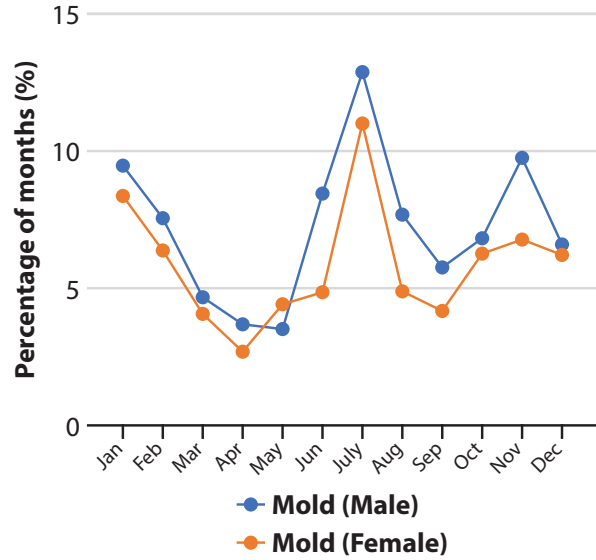

Figure 6. Seasonality of sensitization to specific allergens. (A) the number of patients with common ragweed allergy is increasing year by year. (B) the number of patients with Artemisia, (C) common ragweed, (D) Humulus, (E) house dust, (F) mold in each month. Blue line, male patients; Red line, female patients. The correlations between serum sIgE and months were analysed by Spearman's rank-order test.

\section{Discussion}

Allergic diseases are inflammatory conditions induced by an immunoglobulin $\mathrm{E}$ ( IgE)-mediated reaction in allergen-sensitized subjects. The allergic inflammation is initiated by allergen molecules cross-linking their corresponding receptor-bound sIgE molecules on the surface of mast cells and basophils. Thus, a higher sIgE reflects a higher level of allergen sIgE present on the mast cell and basophil surface, and the amount of sIgE in the serum reflects the degree of an individual's sensitivity to the antigen. ${ }^{10,11}$

Analysis of demographic data of 28879 patients with serum positive allergen sIgEs to variety of common sensitizing allergens indicated that there was a significant gender difference in sensitization to the allergens in patients under 16 years of age ( $\leq 5,6-10$ and $11-15$ years old groups), and significantly greater numbers of males were sensitized compared to females. In terms of age, allergen-positive patients were significantly higher in the 21-30 years and 31-40 years old groups compared to other age groups. For different allergens, house dust sIgE-positive patients were mainly distributed in 11-15 years and 16-20 years old groups and mold sIgE-positive patients in $<5$ years and 6-10 years old groups.
These finding are in accordance with findings of several studies, ${ }^{12,13}$ which showed that younger patients were more sensitized than older patients, and serum sIgE was significantly greater in men than in women. Indeed, a study by Bartra and colleagues has demonstrated that the prevalence of Alternaria sensitization is significantly higher in children compared to adults, and fungal allergy, in particular Alternaria allergy, is a major risk factor for asthma and severe asthma, especially in children presenting genetic condition. ${ }^{14}$ However, it is presently not clear whether the differences in age- and gender-associated sensitization are due to tolerance of the immune system or reflect different levels of exposure to allergen, and needs to be investigated further.

The present study has also demonstrated that Artemisia was the most prevalent sensitizing aeroallergen (41.24\%) in the 28879 patients with positive serum sIgEs, and that the Artemisia grass allergen sIgE was present at mostly level 5 in these patients. Furthermore, the positive rates of Artemisia grass allergen were significantly higher in August and September than in other months of the year. This is consistent with the findings that Artemisia, a common grass in Beijing, 
which flowers in August and September can release a large amount of pollen allergens and lead to seasonal allergic reactions. ${ }^{15}$ These findings are also in accordance with other studies, which have reported sensitization to pollens from grass to be the most prevalent in patients with allergic disease, ${ }^{16,17}$ and have a seasonal onset. ${ }^{18}$ In fact seasonal allergic rhinitis is being the most common disease in our hospital. Similarly, the present study has indicated that Humulus and common ragweed, which are also common grasses in Beijing, are likely to play an important role in sensitizing individuals and impacting their quality of life. Furthermore, we found that annual sensitization to common ragweed showed an increasing trend. Common ragweed is a malignant invasive grass in China, ${ }^{19}$ this change suggests that the spread of ragweed needs to be controlled in time.

In this study, dust mites were found to be the second most sensitizing inhalant allergen in Beijing, with a sensitization rate of $30.89 \%$. This is much lower than the rate of $65 \%$ sensitization to dust mites allergens reported in southern China, ${ }^{20}$ and possibly may be as a consequence of the lower humidity and temperature in Beijing than in southern China. ${ }^{20}$ Similarly, cat allergen was the third most common inhaled allergen is likely to be related to the generally increased number of urban cats and treatment of cats as the most common indoor pets. ${ }^{21}$ This study found that the incidence of sIgE to house dust; an indoor inhalant allergen consisting microorganisms, insects, skin flakes, hair, and fibers; ${ }^{22}$ was higher in May, which might be related to the climate in Beijing.

In this study, we found that the most common food allergens in Beijing were crab, marine fishes, and peanut, which is in accordance with the study of Wang and colleagues. ${ }^{23}$ Furthermore, the percentage of cow's milk and egg sIgE-positive patients were mainly distributed in the $<5$ years old group and gradually decreased with increasing age. This result is in accordance with a recent study, which has demonstrated that production of allergen-specific IgE by food molecular components tend to reduce with ageing. ${ }^{24}$ Some studies have considered the deficiency of gastrointestinal mucosal immune function in infants and the sensitization caused by the combination of macromolecular allergens and gastrointestinal antibodies. ${ }^{25,26}$ Interestingly, our study has demonstrated that crab and marine fishes sIgE-positive patients increase with increasing age after six years old, indicating the effect of different dietary habits on food allergies, which has been demonstrated in different countries and regions. ${ }^{27}$

Our study has indicated that $46.38 \%$ patients (male $44.98 \%$, female $47.98 \%$ ) were sensitized to a single allergen, and $53.62 \%$ patients were sensitized to multiple allergens. The proportion of patients with multiple allergens sensitivity was higher than that of patients with single allergen before 31-40 years old, but after 31-40 years old the proportion of patients with single allergen was higher than that of patients with multiple allergens. These results were consistent with previous studies. ${ }^{28}$ These results may be related to environmental pollution, early childhood allergen exposure, and food supplementation. ${ }^{29}$ In adults, immune tolerance after repeated allergen exposure or food intake resulted in fewer allergens. ${ }^{30}$
Studies have confirmed cross-reactivity between allergens. ${ }^{31}$ Assessment of associations between allergens in the current study has demonstrated that tree allergens are strongly correlated with common ragweed and Humulus allergens, while common ragweed allergen is moderately correlated with Humulus and Artemisia allergens. Similarly, in the case of food allergens, beef and cow's milk, beef and lamb, and cow's milk and egg allergens show a strong correlation, whereas crab and marine fishes, crab and shrimp/prawn, and Soybean and Peanut allergens show moderate correlation. Indeed, there is evidence that the major allergenic protein of Artemisia and common ragweed have a high level of homology, especially Art v1. ${ }^{32}$ Crab, Shrimp/prawn and lobster have cross reacting antigens such as tropomyosin (TM) and arginine kinase $(\mathrm{AK}){ }^{33,34}$ Indeed, TM and $\mathrm{AK}$ allergenic proteins are present in various crustaceans and mollusks such as scallops, Blattel$l a$ and dust mites, and exhibit cross-reactivity among different species. ${ }^{35}$ However, dust mites did not show an association with crab or other allergens in the present study. The correlation between allergens may be related to the close taxonomic relationship. ${ }^{36}$ In contrast, although no significant association was demonstrated between plant pollen and food in the present study, a recent review by Saunders and Platt ${ }^{36}$ has indicated that some pollen-allergic patients develop allergic reaction in the maxillofacial within $2 \sim 15$ min after ingesting fresh plant foods (fruits and vegetables), which is an IgE-mediated allergic reaction to food. ${ }^{37}$ Collectively, these studies suggest that the cross-reactivity between allergens is likely to involve complex mechanisms and further prospective studies and molecular biology experiments are needed to investigate and confirm associations between allergens. For example, longterm follow-up of patients with cross-allergens and proteomic detection of cross-reactive allergens.

In conclusion, this study presented new information about the prevalence of sensitization to different allergens in Beijing, the most inhaled sensitizing allergens were Artemisia grass, and the number of patients with ragweed grass allergy was increasing year by year. These results suggest that grass pollen allergens are becoming the most important allergens in Beijing. Although the incidence of allergic diseases in the Chinese population has previously been documented based on mostly self-reporting questionnaires, ${ }^{4,23}$ this large-scale study reports objectively determined sensitization rates to different allergens among patients with suspected allergic diseases in Beijing, China. The findings of this study may also be applicable to other provinces in northern China, with similar climatic conditions and lifestyles of patients; however, these findings are somewhat limited. First of all, a limited number of allergens were included and some common allergens, such as cypress and birch pollen, were omitted. Second, this was a single-center retrospective chart review study. Thus, confirmation of these findings in large scale prospective multi-center studies to determine the inci $\neg$ dence and prevalence of allergen sensitization in Beijing and different regions of China may help in the prevention, diagnosis, and management of allergic diseases in China. 


\section{Funding}

This work was supported by grants from Beijing municipal science and technology project (Z181100001618002), Beijing science and technology commission science and technology planning project (Z191100009119013) and Beijing health bureau program for high level talents (2014-3-017).

\section{Disclosures}

The authors declare that they have no competing interests.

\section{References}

1. Zhang Y, Zhang L. Increasing prevalence of allergic rhinitis in China. Allergy Asthma Immunol Res. 2019;11(2):156-69.

2. Agache I, Deleanu D, Khaltaev N, Bousquet J. Allergic rhinitis and its impact upon asthma--update (ARIA 2008). Romanian perspective. Pneumologia. 2009;58(4):255-8. Romanian.

3. Rondón C, Campo P, Togias A, Fokkens WJ, Durham SR, Powe DG, et al. Local allergic rhinitis: concept, pathophysiology, and management. J Allergy Clin Immunol. 2012;129(6):1460-7.

4. Wang XD, Zheng M, Lou HF, Wang CS, Zhang Y, Bo MY, et al. An increased prevalence of self-reported allergic rhinitis in major Chinese cities from 2005 to 2011. Allergy. 2016;71 (8):1170-80

5. Liu X, Zhang S, Tsai HJ, Hong X, Wang B, Fang Y, et al. Genetic and environmental contributions to allergen sensitization in a Chinese twin study. Clin Exp Allergy. 2009;39(7):991-8.

6. Campbell DE, Boyle RJ, Thornton CA, Prescott SL. Mechanisms of allergic disease - environmental and genetic determinants for the development of allergy. Clin Exp Allergy. 2015;45(5):844-58.

7. Su KW, Patil SU, Stockbridge JL, Martin VM, Virkud YV, Huang JL, et al. Food aversion and poor weight gain in food protein-induced enterocolitis syndrome: A retrospective study. J Allergy Clin Immunol. 2020;145(5):1430-37.e11.

8. Han YY, Forno E, Gogna M, Celedón JC. Obesity and rhinitis in a nationwide study of children and adults in the United States. J Allergy Clin Immunol. 2016;137(5):1460-5.

9. Bao Y, Chen J, Cheng L, Guo Y, Hong S, Kong W, et al. Chinese Guideline on allergen immunotherapy for allergic rhinitis. J Thorac Dis. 2017;9(11): 4607-50.

10. Jeon H, Jung JH, Kim Y, Kwon Y, Kim ST. Allergen microarrays for in vitro diagnostics of allergies: Comparison with ImmunoCAP and AdvanSure. Ann Lab Med. 2018;38(4):338-47.

11. Chen X, Zheng P, Wei N, Huang H, Luo W, Lin Y, et al. Comparative analysis of serum allergen sIgE detected by western blotting and fluorescent enzyme immunoassay. Clin Lab. 2017;63(3):487-93.

12. Lee JE, Ahn JC, Han DH, Kim DY, Kim JW, Cho SH, et al. Variability of offending allergens of allergic rhinitis according to age: optimization of skin prick test allergens. Allergy Asthma Immunol Res. 2014;6:47-54.

13. Assarehzadegan MA, Shakurnia A, Amini A. The most common aeroallergens in a tropical region in Southwestern Iran. World Allergy Organ J. 2013;6:7.

14. Bartra J, Belmonte J, Torres-Rodriguez JM, Cistero-Bahima A. Sensitization to Alternaria in patients with respiratory allergy. Front Biosci (Landmark edition). 2009;14:3372-9.

15. Ouyang Y, Li J, Zhang D, Fan E, Li Y, Zhang L. A model to predict the incidence of allergic rhinitis based on meteorological factors. Sci Rep. 2017;7(1):10006.

16. Ouyang Y, Yin Z, Li Y, Fan E, Zhang L. Associations among air pollutants, grass pollens, and daily number of grass pollen allergen-positive patients: a longitudinal study from 2012 to 2016. Int Forum Allergy Rhinol. 2019;9(11):1297-303.
17. Lou H, Ma S, Zhao Y, Cao F, He F, Liu Z, et al. Sensitization patterns and minimum screening panels for aeroallergens in self-reported allergic rhinitis in China. Sci Rep. 2017;7(1):9286.

18. Blanca-Lopez N, Campo P, Salas M, Rodríguez CG, Palomares F, Blanca $\mathrm{M}$, et al. Seasonal local allergic rhinitis in areas with high concentrations of grass pollen. J Invest Allergol Clin Immunol. 2016;26(2):83-91.

19. Li J, Sun B, Huang Y, Lin X, Zhao D, Tan G, et al. A multicentre study assessing the prevalence of sensitizations in patients with asthma and/or rhinitis in China. Allergy. 2009;64:1083-92.

20. Zheng YW, Li XX, Zhao DY, Zhang CQ, Chen JJ, Zhang Luo, et al. Indoor allergen levels and household distributions in nine cities across China. Biomed Environ Sci. 2015;28:709-17.

21. Ownby DR, Johnson CC, Peterson EL. Exposure to dogs and cats in the first year of life and risk of allergic sensitization at 6 to 7 years of age. JAMA. 2002;288(8):963-72.

22. Araki A, Ait Bamai Y, Ketema RM, Kishi R. House dust and its adverse health effects. Nihon Eiseigaku Zasshi. Jap J Hyg. 2018;73(2):130-7. Japanese.

23. Wang XY, Zhuang Y, Ma TT, Zhang B, Wang XY. Prevalence of self-reported food allergy in six regions of inner mongolia, Northern China: a population-based survey. Med Sci Monit. 2018;24:1902-11.

24. Tosca MA, Silvestri M, Olcese R, Sacco O, Pistorio A, Rossi GA, et al Allergen-specific IgE to food molecular components and age: From early childhood to adulthood. Allergol Immunopathol (Madr). 2017;45(1):87-92.

25. Kau AL, Ahern PP, Griffin NW, Goodman AL, Gordon JI. Human nutrition, the gut microbiome and the immune system. Nature. 2011;474: 327-36.

26. Virta LJ, Kautiainen H, Kolho KL. Symptoms suggestive of cow's milk allergy in infancy and pediatric inflammatory bowel disease. Pediatr Allergy Immunol. 2016;27(4):361-7.

27. Gelincik A, Büyüköztürk S, Gül H, Ișik E, Isssever $H$, Ozşeker F et al. Confirmed prevalence of food allergy and non-allergic food hypersensitivity in a Mediterranean population. Clin Exp Allergy. 2008, 38(8):1333-41.

28. Hong SN, Won JY, Nam EC, Kim TS, Ryu YJ, Kwon JW, et al. Clinical manifestations of allergic rhinitis by age and gender: A 12-year single -center study. Ann Otol Rhinol Laryngol. 2020;129:910-7.

29. Strachan DP. Family size, infection and atopy: the first decade of the "hygiene hypothesis". Thorax. 2000;55 Suppl 1:S2-10.

30. Spergel JM. From atopic dermatitis to asthma: the atopic march. Ann Allergy Asthma Immunol. 2010;105:99-106;quiz 7-9,17.

31. Cabanillas B, Jappe U, Novak N. Allergy to peanut, soybean, and other legumes: Recent advances in allergen characterization, stability to processing and IgE cross-reactivity. Mol Nutr Food Res. 2018;62(1).

32. Hirschwehr R, Heppner C, Spitzauer S, Sperr WR, Valent P, Berger U, et al Identification of common allergenic structures in mugwort and ragweed pollen. J Allergy Clin Immunol. 1998;101(2 Pt 1):196-206.

33. Yang Y, Hu MJ, Jin TC, Zhang YX, Liu GY, Li YB, et al. A comprehensive analysis of the allergenicity and IgE epitopes of myosinogen allergens in Scylla paramamosain. Clin Exp Allergy. 2019;49(1):108-19.

34. Pedrosa M, Boyano-Martínez T, García-Ara C, Santiago Q. Shellfish allergy: a comprehensive review. Clin Rev Allergy Immunol. 2015;49(2): 203-16.

35. Abdel Rahman AM, Kamath SD, Lopata AL, Robinson JJ, Helleur RJ. Biomolecular characterization of allergenic proteins in snow crab (Chionoecetes opilio) and de novo sequencing of the second allergen arginine kinase using tandem mass spectrometry. J Proteomics. 2011; 74(2):231-41.

36. Ji Q, Xu X, Wang K. Genetic transformation of major cereal crops. Int J Dev Biol. 2013;57(6-8):495-508.

37. Saunders S, Platt MP. Oral allergy syndrome. Curr Opin Head Neck Surg. 2015;23(3):230-4. 
Supplementary Table 1. Age and sex distribution in 28879 sIgE positive patients. Significance of gender and age differences among patients who tested positive for sIgE in each age group was analysed by chi-square $\left(\chi^{2}\right)$ analysis. $n$, number of cases.

\begin{tabular}{|c|c|c|c|c|c|c|}
\hline $\begin{array}{c}\text { Age } \\
\text { (years) }\end{array}$ & $\begin{array}{l}\text { Positive } \\
\text { cases (n) }\end{array}$ & $\begin{array}{c}\text { Percentage } \\
(\%)\end{array}$ & $\begin{array}{l}\text { Male Positive cases } \\
\text { (n/\%) }\end{array}$ & $\begin{array}{l}\text { Female Positive cases } \\
\qquad(\mathbf{n} / \%)\end{array}$ & $x^{2}$ & $P$ \\
\hline$\leq 5$ & 1840 & $6.37 \%$ & $1202(56.22 \%)$ & $638(47.86 \%)$ & 23.03 & 0.00 \\
\hline $6-10$ & 3453 & $11.96 \%$ & $2182(67.22 \%)$ & $1271(61.49 \%)$ & 18.23 & 0.00 \\
\hline $11-15$ & 2073 & $7.18 \%$ & $1285(68.42 \%)$ & $788(62.39 \%)$ & 12.24 & 0.00 \\
\hline $16-20$ & 1430 & $4.95 \%$ & $806(59.09 \%)$ & $624(59.15 \%)$ & 0.00 & 0.98 \\
\hline $21-30$ & 6254 & $21.66 \%{ }^{* *}$ & $3023(52.48 \%)$ & $3231(52.23 \%)$ & 0.08 & 0.78 \\
\hline $31-40$ & 7256 & $25.13 \% * *$ & $3629(48.73 \%)$ & $3627(48.63 \%)$ & 0.02 & 0.90 \\
\hline $41-50$ & 3295 & $11.41 \%$ & $1636(40.35 \%)$ & $1659(41.21 \%)$ & 0.61 & 0.44 \\
\hline $51-60$ & 2209 & $7.65 \%$ & $1070(35.8 \%)$ & $1139(34.76 \%)$ & 0.76 & 0.38 \\
\hline$\geq 61$ & 1069 & $3.7 \%$ & $558(31.56 \%)$ & $511(29.22 \%)$ & 2.28 & 0.13 \\
\hline Total & 28879 & $100 \%$ & $15391(53.29 \%)$ & $13488(46.71 \%)$ & 44.86 & 0.00 \\
\hline
\end{tabular}

